Original Research Paper

\title{
Performance Evaluation of Interference Mitigation Techniques in 4G Networks in Mobile Environments
}

\author{
${ }^{1}$ Emna Hajlaoui, ${ }^{1}$ Abdelhakim Khlifi, ${ }^{2}$ Samia Dardouri, \\ ${ }^{2}$ Aida Zaier, ${ }^{1}$ Mouna Ben Hamed and ${ }^{1}$ Lassâad Sbita \\ ${ }^{1}$ National Engineering School of Gabes (ENIG), University of Gabes, Tunisia \\ ${ }^{2}$ National Engineering School of Tunis (ENIT), University of Carthage, Tunisia
}

\section{Article history}

Received: 01-08-2019

Revised: 14-10-2019

Accepted: 24-12-2019

Corresponding Author:

Emna Hajlaoui

National Engineering School of

Gabes (ENIG), University of

Gabes, Tunisia

Email: emhajlaoui@yahoo.fr

\begin{abstract}
Intercell Interference (ICI) is one of the major factors that limit the performance and Quality of Service (QoS) of 4G wireless networks (Long Term Evolution/LTE-Advanced). For this reason, heterogeneous networks present an attractive solution for the improvement of mobile network's services to provide better data rates and coverage. So, a key scheme in $4 \mathrm{G}$ traffic processing is the interference mitigation techniques. In the present work, we propose a model while optimizing the simulation parameters of three well-known ICI mitigation algorithms, namely Soft Frequency Reuse (SFR), Distributed Fractional Frequency Reuse (DFFR) and Strict Frequency Reuse (Strict FR). Here, mobile users are considered to be moving at high speed $(300 \mathrm{Km} / \mathrm{h}$ : speed allowed by the LTE/LTE-A standards). We provide a simulation framework that is suitable for the parameters of the $4 \mathrm{G}$ system in terms of cells number and inter-eNodeB distance for an LTE/LTE-A network deployed in an urban area. Subsequently, we compare our proposed model with the aforementioned algorithms in terms of throughput, Signal-toInterference-plus-Noise-Ratio (SINR) and Spectral efficiency by using the NS3 open source simulator. Results prove the efficiency of the proposed model in condensed area of mobile users compared to previous works that evaluated and tested immobile scenarios.
\end{abstract}

Keywords: Intercell Interference, 4G, Mitigation Techniques, SINR, Throughput, Spectral Efficiency

\section{Introduction}

The third Generation Partnership Program (3GPP) members started a feasibility study on the enhancement of the Universal Terrestrial Radio Access (UTRA) in the aim of continuing the long time frame competitiveness of the 3G Universal Mobile Telecommunications System (UMTS) technology beyond High Speed Packet Access (HSPA) (Veeresh et al., 2018; Pomilio de Oliveira et al., 2018; Harwahyu et al., 2016). This project is called Long Term Evolution (LTE/LTE-A) (3GPP TS 25.913, 2009; Veeresh et al., 2018; Pomilio de Oliveira et al., 2018; Harwahyu et al., 2016). In fact, LTE/LTE-A is a fourth generation $(4 \mathrm{G})$ cellular network designed to meet the demand for higher data rates in mobile communication (Veeresh et al., 2018; Pomilio de Oliveira et al., 2018; Harwahyu et al., 2016; Zaier et al., 2016; Hussain, 2009; Ghassasn et al., 2012). One of the benefits of LTE/LTE-A systems is the support of heterogeneous networks that allow the coverage of the vast macro site without investing in expensive macrocell infrastructure. In fact, $4 \mathrm{G}$ systems (LTE/LTE-A) will bring many technical benefits to cellular networks. Bandwidth will be scalable from $1.25 \mathrm{MHz}$ to $20 \mathrm{MHz}$ (Zaier et al., 2016; Ghassasn et al., 2012). This will suit the needs of different network operators that have different bandwidth allocations and also allow operators to provide diverse services based on spectrum (Veeresh et al., 2018; Pomilio de Oliveira et al., 2018; Harwahyu et al., 2016; Zaier et al., 2016). LTE/LTE-A networks have set aggressive performance requirements that rely on physical layer technologies, such as Orthogonal Frequency Division Multiplexing (OFDM), Single Carrier Frequency Division Multiplexing Access (SCFDMA), Multiple-Input Multiple-Output (MIMO) systems and Smart Antennas to achieve these targets (Zaier et al., 2016; Holma and Toskala, 2009). Thus, the main objectives of $4 \mathrm{G}$ networks are to minimize the 
system and User Equipment (UE) complexities, allow flexible spectrum deployment in existing or new frequency spectrum and to ensure co-existence with other 3GPP Radio Access Technologies (RATs) (Zaier et al., 2016; Holma and Toskala, 2009; Dikamba, 2011; Singh and Singh, 2013). In this context, the efficiency of $3 \mathrm{G}$ networks allow carriers to provide more data and voice services over a given bandwidth. For this reason, OFDM and SCFDMA have been applied in the physical layer of LTE/LTE-A uplink systems thanks to their high data transmission rate and high bandwidth efficiency to mitigate the Inter-Symbol Interference (ISI) in a severe multi-path fading channel (Zaier et al., 2016; Holma and Toskala, 2009; Dikamba, 2011; Singh and Singh, 2013; Sadiq et al., 2009). In fact, the interference issue becomes severer for heterogeneous LTE/LTE-A networks, where the low-power nodes such as pico/femto-cells are deployed within the coverage area of a macro-cell network to improve the coverage and spectrum efficiency (Sbit et al., 2018). Although the LTE/LTE-A specs describe both Frequency Division Duplex (FDD) and Time Division Duplex (TDD) to separate Uplink and Downlink traffic, market preferences dictate that the majority of deployed systems are FDD (Zaier et al., 2016; Sbit et al., 2018). In addition, scheduling is also an extremely important factor and is a key Radio Resource Management (RRM) mechanism for realizing Quality of Service (QoS) requirements and optimizing system performance of LTE/LTE-A networks. As specified, the radio network will be optimized for higher performance. Furthermore, LTE/LTE-A standards are designed for single-frequency deployment, that is, each Base Station (BS) uses the entire frequency spectrum for transmissions. While the aggressive reuse of frequencies could provide higher system capacity, the strong Intercell Interference (ICI) from other cells results in Signal-to-Interference-plus-Noise-Ratio (SINR) degradation for cell-edge users, which severely limits the cell-edge spectrum efficiency (Sbit et al., 2018). In addition, to meet the traffic demand in a hot spot area with numerous users "such as stadiums, airports, office buildings..." a dense deployment and spectrum reuse are necessary to have a significant ICI (Manli et al., 2015).

Against this background and in order to achieve high-data wireless transmissions, dense reuse of available frequency spectrum and heterogeneous deployment are envisaged for LTE/LTE-A systems. Such features make ICI mitigation more challenging than before (Manli et al., 2015).

In this context, ICI is one of the major factors that limit the performance of $4 \mathrm{G}$ wireless networks. Scheduling algorithms such as Fractional Frequency Reuse (FFR), Soft Frequency Reuse (SFR), Strict
Frequency Reuse (Strict FR) and Distributed Fractional Frequency Reuse (DFFR), have been introduced as an effective way to optimize spectrum efficiency and reduce ICI in LTE/LTE-A networks (Manli et al., 2015; Giménez et al., 2015). Furthermore, some models and simulations have been proposed to improve the $4 \mathrm{G}$ system performances, especially in terms of SINR, throughput and cell spectral efficiency (Manli et al., 2015; Giménez et al., 2015; Noor Shahida et al., 2015; Hung-Chin and Wei-Di, 2015; Slawomir, 2017; Sinh Cong et al., 2015; Asif et al., 2017; Christos et al., 2013). In this work, we propose a model while optimizing the simulation parameters of the studied models in order to cancel the maximum possible ICI.

The rest of the paper is organized as follows. Section 2 briefly provides quick overview of related works on evaluating interference mitigation techniques in $4 \mathrm{G}$ networks. Section 3 outlines the interference in LTE/LTE-A networks. Section 4 introduces the mitigation techniques in $4 \mathrm{G}$ systems. Our system model is presented in section 5. Section 6 introduces the simulation environment by presenting the used simulator, the performance indexes of our simulations as well as the simulation parameters. Section 7 interprets the simulation results generated using the open source network simulator NS3. Finally, the conclusion of this paper is presented in section 8 .

\section{Related Works}

To meet the QoS demands for different applications, many packet-scheduling algorithms have been developed to allocate limited frequency and time resources efficiently and fairly to real-time and non-realtime traffic for all data transfer devices including mobile and wireless networks. Indeed, ICI is one of the major factors that limit the performance of $4 \mathrm{G}$ wireless networks. Scheduling algorithms such as Fractional Frequency Reuse (FFR), Soft Frequency Reuse (SFR), Strict Frequency Reuse (Strict FR) and Distributed Fractional Frequency Reuse (DFFR), have been introduced as an effective way to optimize spectrum efficiency and reduce ICI in LTE/LTE-A networks (Manli et al., 2015; Giménez et al., 2015). In fact, application of fractional frequency reuse in the LTE/LTEA systems has been a highly-discussed topic in communication society (Manli et al., 2015; Giménez et al., 2015; Noor Shahida et al., 2015; Hung-Chin and Wei-Di, 2015; Slawomir, 2017; Sinh Cong et al., 2015; Asif et al., 2017; Christos et al., 2013). However, the comparison between these algorithms has not been fully presented by the researchers proposing the models. In fact, some models and simulations have been proposed to improve the $4 \mathrm{G}$ system performances, especially in terms of SINR, throughput and cell spectral efficiency (Manli et al., 2015; Giménez et al., 2015; Noor Shahida et al., 
2015; Hung-Chin and Wei-Di, 2015; Slawomir, 2017; Sinh Cong et al., 2015; Asif et al., 2017; Christos et al., 2013). The proposed models use different simulation parameters to cancel the maximum possible ICI. Therefore, different transmit eNodeB powers have been assumed. In another paper, they have modified inter-eNodeB distance of the $4 \mathrm{G}$ system in order to minimize ICI (Manli et al., 2015; Giménez et al., 2015; Noor Shahida et al., 2015; Hung-Chin and Wei-Di, 2015; Slawomir, 2017; Sinh Cong et al., 2015; Asif et al., 2017; Christos et al., 2013). Cell-layout, number of users and bandwidth are also taken into account in order to mitigate ICI. However, most of these authors did not take into account the effect of user's distribution. Indeed, the majority of these studies have assumed that users in the considered cells are immobile in the concerned cells. In this work, we propose a model while optimizing the simulation parameters of the studied models. Therefore, the contribution of this paper that distinguishes it from these works is summarized in the following points:

The major contribution of this work is to consider mobile users moving at $300 \mathrm{Km} / \mathrm{h}$ (speed allowed by the LTE/LTE-A standards).

Simulation parameters of the studied methods were optimized in order to have a model that is compliant with the parameters of LTE/LTE-A standards (we provide a simulation framework that is compliant with the parameters of the $4 \mathrm{G}$ system).

We consider the three well-Known ICI mitigation algorithms were considered: SFR, DFFR and Strict FR. Subsequently, we compare the proposed model with the aforementioned algorithms in terms of throughput, SINR and Cell spectral efficiency in different scenarios.

\section{Interference in Lte/Lte-A Networks}

ICI presents a major challenge in $4 \mathrm{G}$ networks (LTE and LTE-A) (Sbit et al., 2018). Indeed, because of the increasing numbers of cells or users, disturbances and interference between the cells of the $4 \mathrm{G}$ networks have grown in a troublesome way (Manli et al., 2015; Giménez et al., 2015; Noor Shahida et al., 2015; Hung-Chin and Wei-Di, 2015; Slawomir, 2017; Sinh Cong et al., 2015; Asif et al., 2017; Christos et al., 2013). Therefore, cells interfere with neighboring or adjacent ones, which cause poor transmission or reception of data Fig. 1.

In fact, the problem of intracellular interferences in $4 \mathrm{G}$ networks is the problem of scrambling between the different entities of the same cell as indicates the name Fig. 2. This is essentially due to the increase in the number of users. While the Orthogonal Frequency Division Multiple Access (OFDMA) is the multiple access technique chosen by 3GPP for the downlink of the radio interface of $4 \mathrm{G}$ systems, the data is transmitted on a large number of parallel subcarriers and narrow bands. Since the subcarriers are orthogonal, the intracellular interference can be eliminated.

However, ICI remains the major problem for $4 \mathrm{G}$ networks. It limits the performance of the system and reduces the achievable throughput, especially for the users' equipment located on the edge of the cells. Whose users experience high ICI (Manli et al., 2015; Giménez et al., 2015; Noor Shahida et al., 2015; Hung-Chin and Wei-Di, 2015; Slawomir, 2017; Sinh Cong et al., 2015; Asif et al., 2017; Christos et al., 2013).

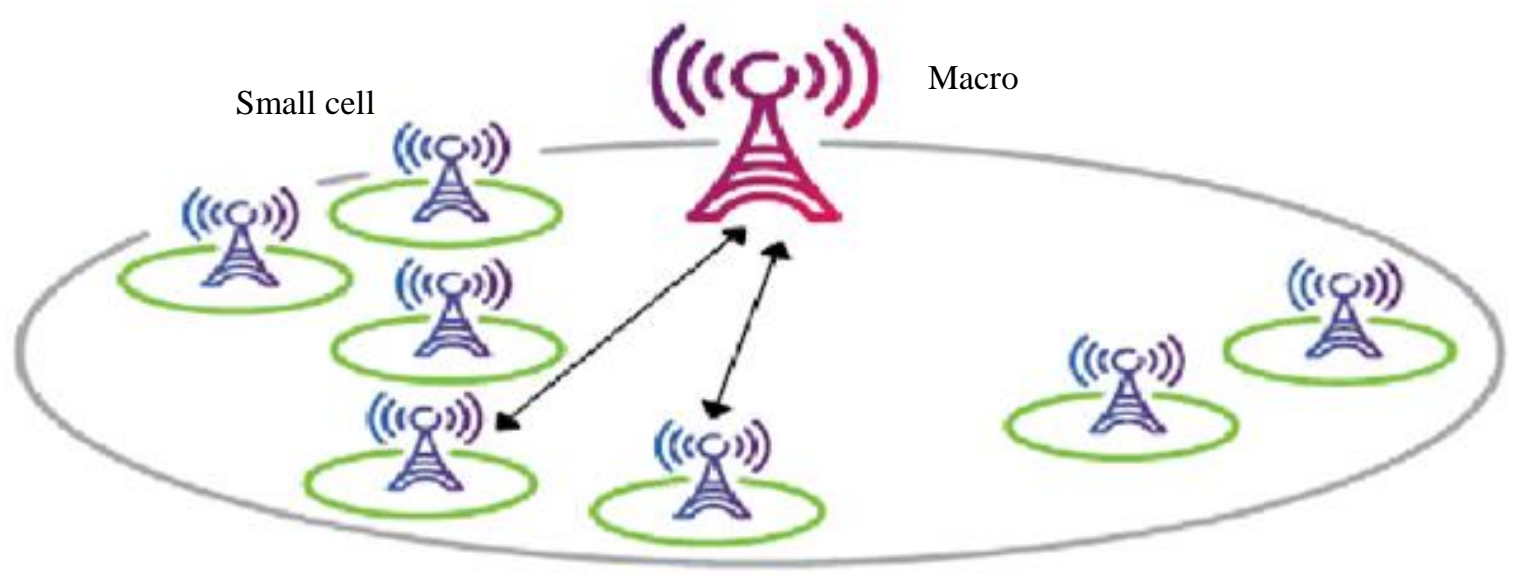

Fig. 1: Intercell interference in $4 \mathrm{G}$ networks 


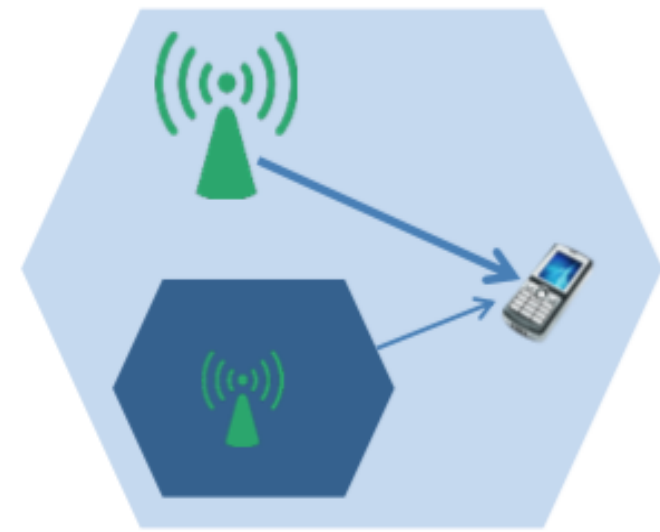

Fig. 2: Intracellular interference in $4 \mathrm{G}$ networks

\section{Mitigation Techniques in $\mathbf{4 g}$ Networks}

In order to achieve high QoS for subscribers, it is necessary to find network interference avoidance solution. Against this background, the method "Dense Frequency reuse" makes it possible to use frequencies with the aim of improving the capacity of the system by increasing the number of RBs available in each cell Fig. 3. It is a necessary for mobile network operators seeking to meet the huge data needs, due to the proliferation of mobile applications and the exponential increase in the number of connected devices, to find obvious solutions to overcome the undesirable effects of interference.

A wide variety of interference mitigation techniques have been proposed in the literature. For example, Frequency reuse is one of the techniques that is adopted in $4 \mathrm{G}$ networks to mitigate ICI and allow the given frequency band to be reused fully for each cell of the network (Singh and Singh, 2013; Sadiq et al., 2009; Sbit et al., 2018; Manli et al., 2015; Giménez et al., 2015; Noor Shahida et al., 2015; Hung-Chin and Wei-Di, 2015; Slawomir, 2017;) Fig. 3. However, frequency bands intersecting between adjacent cells, mostly at the cell edge of the network, cause ICI. To overcome this problem, FFR as well as the DFFR techniques were introduced in the $4 \mathrm{G}$ systems Fig. 4.

Indeed, FFR and SFR are two spectrum reuse methods that have been proposed to improve spectrum efficiency and reduce ICI in LTE/LTE-A networks Fig. 4. In these two techniques, the subcarriers are divided into a cell center and a cell edge group. The ICI is controlled by setting different power levels for the cell center and cell edge subcarrier groups used by the users in cell center and cell edge regions, respectively (Manli et al., 2015; Giménez et al., 2015; Noor Shahida et al., 2015; Hung-Chin and Wei-Di, 2015; Slawomir, 2017; Sinh Cong et al., 2015; Asif et al., 2017; Christos et al., 2013). However, DFFR technique was proposed as a means to mitigate ICI and improve the overall performance of FFR in terms of coverage and capacity in $4 \mathrm{G}$ wireless networks. In what follows, we focus on three effective ICI mitigation strategies.

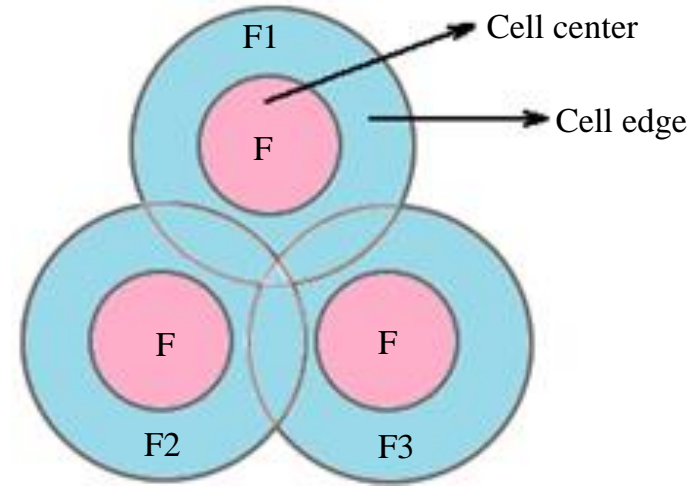

Fig. 3: Frequencey reuse concept

\section{A. Fractional Frequency Reuse Technique}

Fractional Frequency Reuse (FFR) algorithm can be applied as an interference management technique. In FFR schemes, the whole available bandwidth is divided into several sub-bands. Every cell is split into two areas, cell-center and cell-edge, which are forced to use different sub-bands. These are allocated among the cells in such a way that contiguous cells do not use the same sub-band in the cell-edge region. Indeed, in FFR technique, to ensure that mutual interference between users and base stations remain below a harmful level, neighboring cells use different frequencies. Moreover, FFR technique is a compromise between reuse- 1 and reuse-N models. Each cell is divided into cell-center and cell-edge zones, where frequency reuse-1 model is used in the cell-center zone, while a higher frequency reuse factor is used in cell-edge zone. The available spectrum is divided into two sub-bands: The first one is permanently used in cell-center zones, while the second sub-band is used according to frequency reuse- $\mathrm{N}$ model in the cell-edge zones (Manli et al., 2015; Giménez et al., 2015; Noor Shahida et al., 2015; Hung-Chin and Wei-Di, 2015; Slawomir, 2017; Sinh Cong et al., 2015; Asif et al., 2017; Christos et al., 2013).

Figure 5 illustrates an example of an FFR technique.

\section{B. Strict Frequency Reuse Technique}

Strict FR is a modification of the use of traditional frequencies used in multi-cell networks. Figure 6 illustrates an example of Strict FR method for a hexagonal grid deployment. In fact, the users inside each cell are assigned to a common frequency sub-band, while the bandwidth on the cell where the users are onboard is divided up through the cells according to a RF reusability factor. For example, in strict FR scheme (with reuse factor $=3$ ), the whole bandwidth is dived into one common sub-band and three private sub-band group. Consequently, the common sub-bands are allocated to cell-center area with low power level in every cell while each private sub-band group is allocated to the cell-edge area at high power level. 


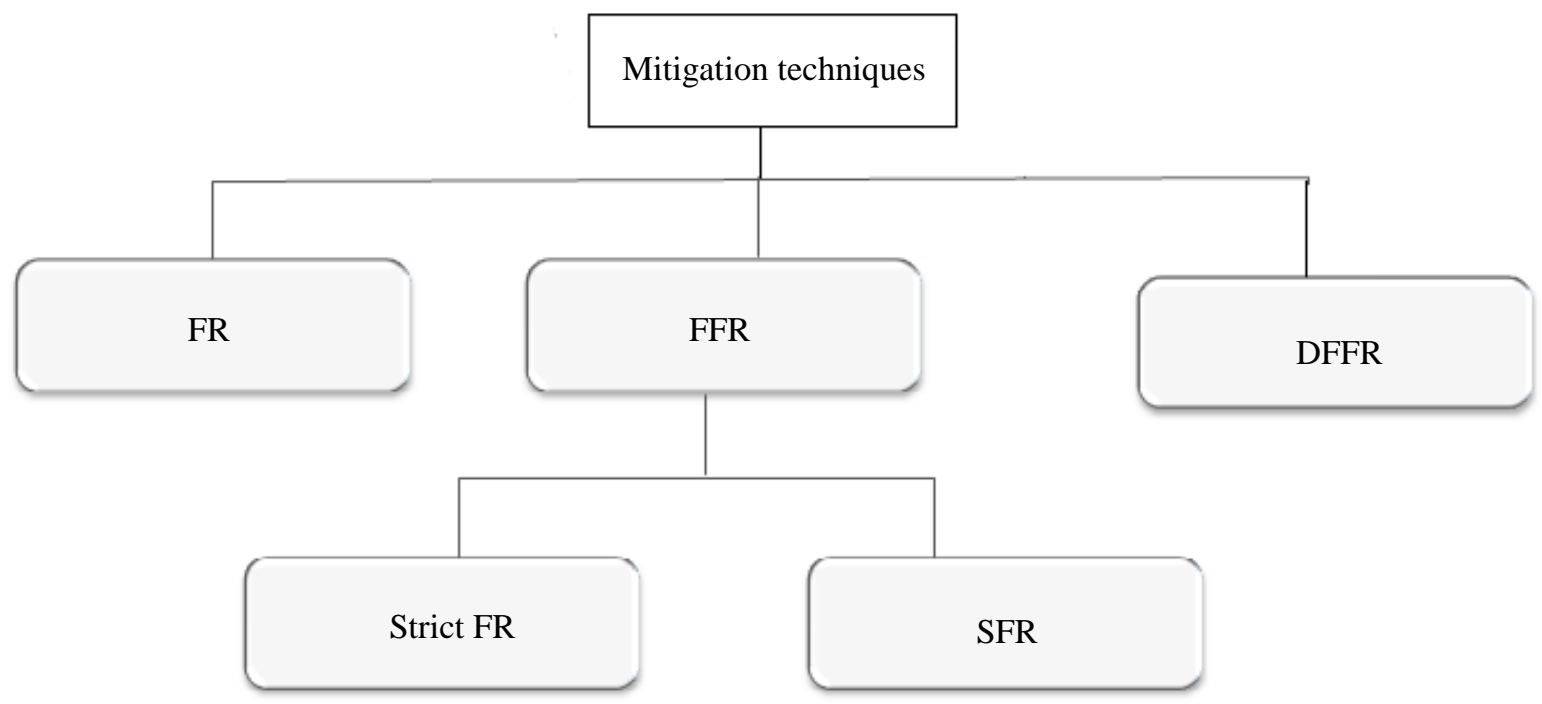

Fig. 4: Mitigation techniques

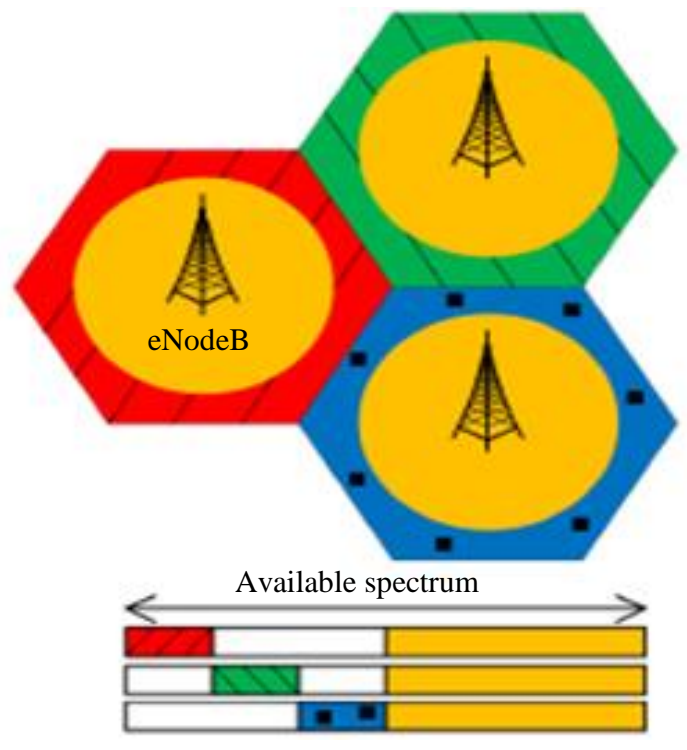

Fig. 5: FFR Scheme

In fact, it is noticed that cell-center users do not share their own sub-bands to the cell-edge users and then a cell-center (cell-edge) user is only affected by interference caused by base station transmitting on the same cell-center (cell-edge) sub-band (Manli et al., 2015; Giménez et al., 2015; Noor Shahida et al., 2015; Hung-Chin and Wei-Di, 2015; Slawomir, 2017; Sinh Cong et al., 2015; Asif et al., 2017; Christos et al., 2013).

\section{Soft Frequency Reuse Technique}

Figure 7 illustrates an SFR deployment. Indeed, an SFR scheme has been proposed as an alternative to the FFR method. The main difference between the FFR and SFR techniques is that the SFR scheme allows the cell center users to use the whole spectrum, while the FFR scheme does not. As a consequence, the SFR technique can achieve good compromise between bandwidth availability and interference.

In fact, in SFR, each cell uses the total available bandwidth. For each sector, cell edge users are allocated in the fraction of bandwidth with the highest power level, but cell center users are allocated with lower power in the rest of the frequency band. SFR scheme is a modification of Strict FR in which the whole bandwidth is divided into two sub-bands, called the cell-edge and cell-center sub-bands. The main difference between Strict and Soft FR is that the cell-center users share the allocated sub-bands with cell-edge users in adjacent cells in an effort to improve spectrum efficiency and system performance (Manli et al., 2015; Giménez et al., 2015; Noor Shahida et al., 2015; Hung-Chin and Wei-Di, 2015; Slawomir, 2017; Sinh Cong et al., 2015; Asif et al., 2017; Christos et al., 2013).

\section{Distributed Fractional Frequency Reuse Technique}

DFFR was proposed as a means to mitigate ICI and improve the overall performance of FFR in terms of coverage and capacity in $4 \mathrm{G}$ wireless networks. According to the development of DFFR, users and subcarriers within a cell are divided into two groups, (1) super groups cover all the cells and the subcarriers are allocated and given to any user in the cell and (2) regular groups, which is further partitioned into three sectors. 


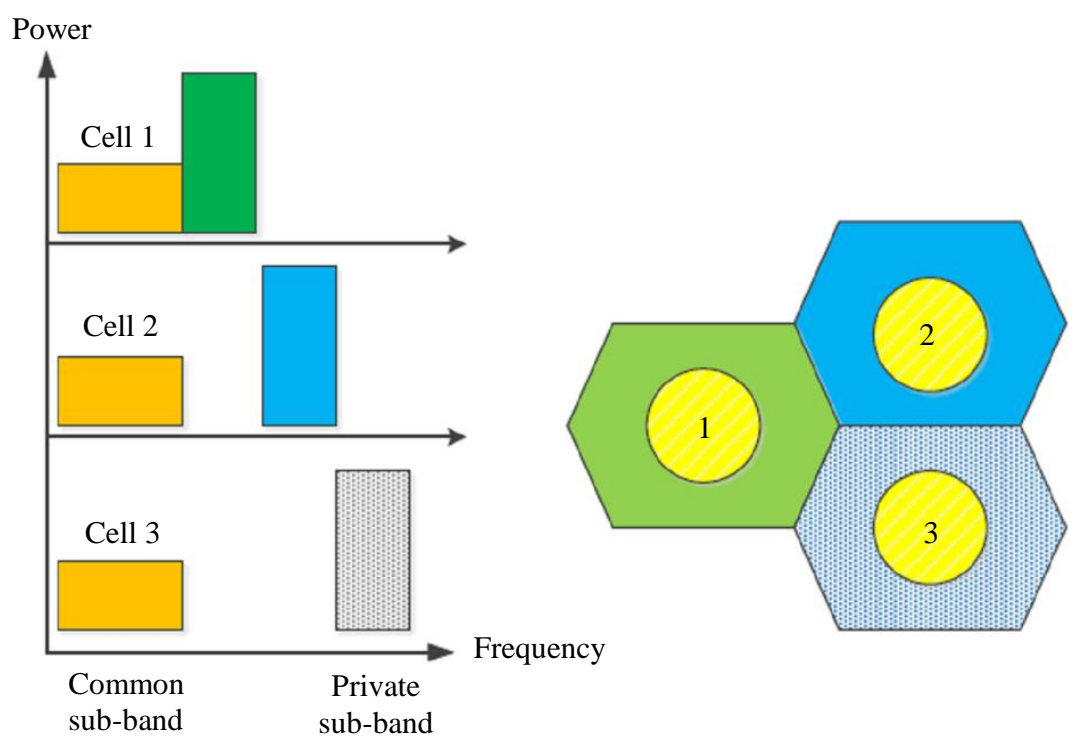

Fig. 6: Strict FR scheme
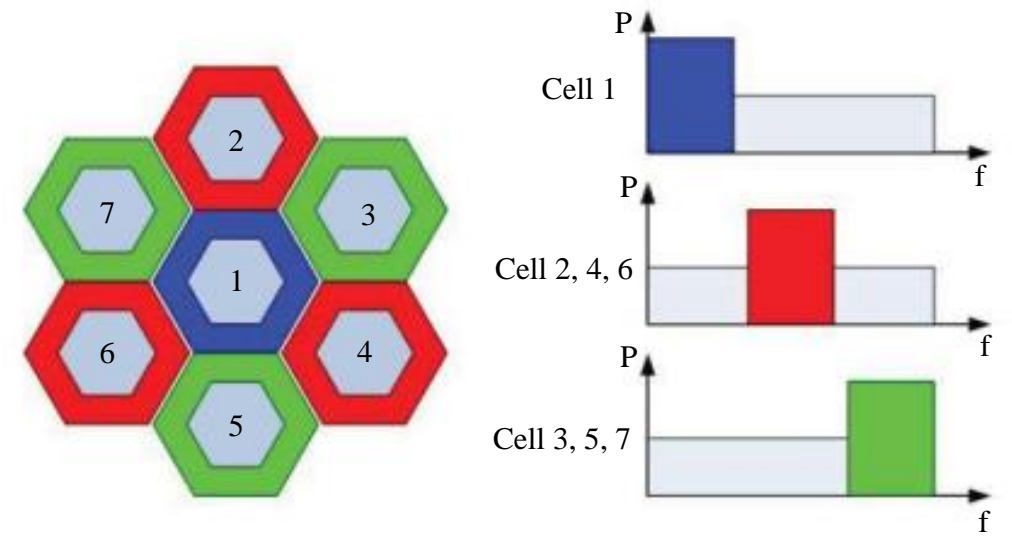

Fig. 7: SFR scheme

\section{System Model}

\section{A. Deployment Model}

In this study, we consider an uplink/downlink transmission in an LTE/LTE-A network. In fact, the system model consists of seven adjacent Macro Base Stations (MBS). MBS coverage is modeled as a sectorized hexagonal layout, where each site consists of three adjacent sectors, each of which consists of seven adjacent cells. In addition, each sector is served by an eNodeB that has its own bandwidth and power allocation policy. Therefore, as mentioned earlier, cell geometry is hexagonal (21 hexagonal cell) and each site consists of three adjacent sectors, where each sector is served by an eNodeB. IntereNodeB distance equals $500 \mathrm{~m}$, which corresponds to an
LTE/LTE-A network deployed in an urban area. To examine the performance of the proposed model compared to the three well-known frequency reuse algorithms, Users Equipment (UE) move at $300 \mathrm{~km} / \mathrm{h}$ Fig. 8.

\section{UE Distribution}

As mentioned before, the major contribution is to consider mobile users moving at $300 \mathrm{Km} / \mathrm{h}$ (speed allowed by the LTE/LTE-A standards) in order to test the pedestrian and vehicular scenarios. We consider UE distribution between cell zones as an essential parameter in the simulations, since it has an important impact on UE throughput and on system performance. UE distribution between cell zones also has a great impact on ICI and consequently on system throughput. 


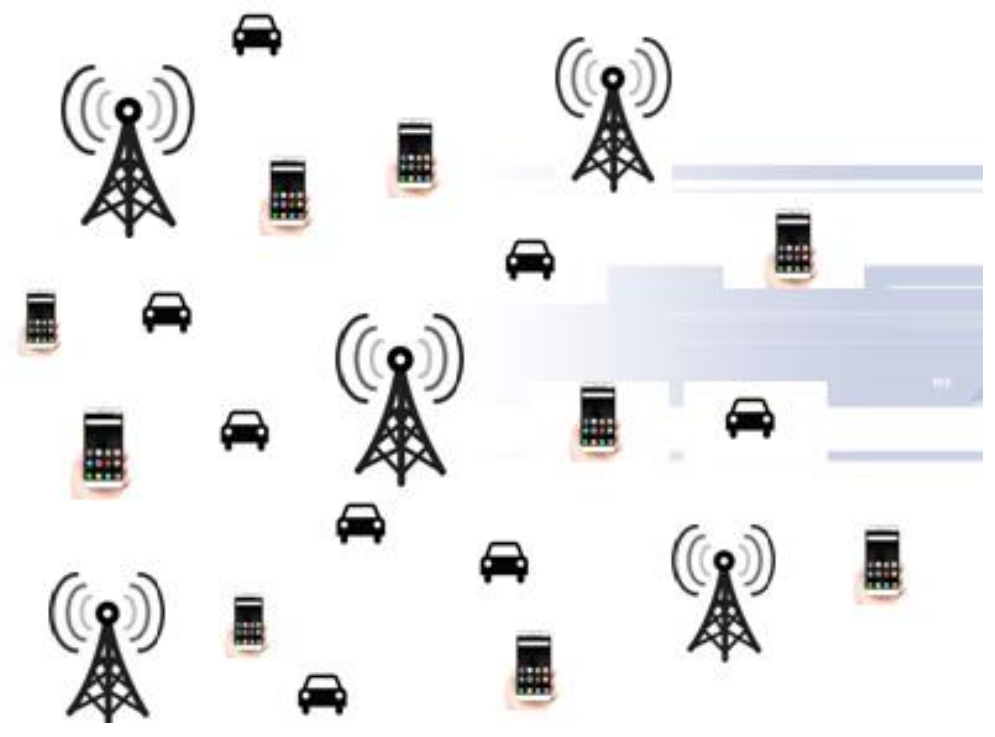

Fig. 8: Simulation network model

\section{Simulation Environment}

The simulations are performed using Network Simulator 3 (NS-3) version 25 which can be used on Linux or Windows platforms. Accordingly, we used Ubunto as the Linux interface to install NS3. In fact, the performance indexes of the simulations were:

- $\quad$ Signal to Interference plus Noise Ratio (SINR)

- Throughput

- Cell Spectral Efficiency

\section{A. Signal to Interfernce plus Noise Ratio (SINR)}

The SINR is defined as follows (Dikamba, 2011):

$$
\operatorname{SINR}_{m}=\frac{\mathrm{S}}{\mathrm{N}+\mathrm{I}}
$$

where, $S$ is the desired signal's power, $N$ is noise's power and $I$ is the total interference's power. With, $S$ and $N$ are expressed by Equations (2) and (3) respectively as (Dikamba, 2011):

$$
S=P_{M} G_{m, M}
$$

where, $P_{M}$ is the transmit power of servant macro base station $G_{m, M}$ and $M$ is the channel gain between the macro user $m$ and the servant macro base station $M$.

$$
N=N_{0} \Delta_{f}
$$

where, $N_{0}$ presents the white noise power spectral density and $\Delta_{f}$ is the subcarrier spacing.

In the simulation, the SINR is calculated in uplink and downlink.

\section{B. Throughput}

The Throughput is defined as follows (Dikamba, 2011):

Throughput $_{m}=W \log _{2}\left(1+\operatorname{SINR}_{m}\right)$

where, $W$ is the available bandwidth.

In the simulation, the throughput is calculated in uplink and downlink.

\section{Spectral Efficiency}

The Spectral Efficiency is defined as follows (Dikamba, 2011):

Spectral Efficiency $=\frac{\text { Throughput }}{W}$

where, $W$ is the available bandwidth.

In the simulation, the Spectral Efficiency is calculated in uplink and downlink.

\section{Simulation Parameters}

As we mentioned before, ICI mitigation technique has become one of the major concerning issues in the communication sector. In fact, studied models (Manli et al., 2015; Giménez et al., 2015; Noor Shahida et al., 2015; Hung-Chin and Wei-Di, 2015; Slawomir, 2017; Sinh Cong et al., 2015; Asif et al., 2017; Christos et al., 2013) use different simulation parameters to cancel the maximum possible ICI. Therefore, different transmit eNodeB powers have been assumed. In another paper, they have modified inter-eNodeB 
distance of the $4 \mathrm{G}$ system $(500 \mathrm{~m})$ in order to minimize ICI. Cell-layout (21 cells), number of users and bandwidth are also taken into account in order to mitigate ICI. Furthermore, comparison of these different studied models is summarized in Table 1 .

As we remark, most of these works did not take into account the effect of user's distribution. Indeed, the majority of these studies have assumed that users in the considered cells are immobile in the concerned cells. In this work, we propose a model while optimizing the simulation parameters of the studied models. Therefore, the major contribution of this paper is to consider mobile users moving at $300 \mathrm{Km} / \mathrm{h}$ (speed allowed by the LTE/LTE-A standards). In fact, simulation parameters of the studied methods were optimized in order to have a model that is compliant with the parameters of $4 \mathrm{G}$ standards (we provide a simulation framework that is compliant with the parameters of the $4 \mathrm{G}$ system).

Furthermore, in the simulations, we assumed an urban area where the UE was randomly distributed around the sites and join the network automatically at a speed of $300 \mathrm{~km} / \mathrm{h}$ in order to test the pedestrian and vehicular scenarios.

Simulation parameters (compliant with those of the $4 \mathrm{G}$ system) are summarized in Table 2.

Table 1: Comparison of different models

\begin{tabular}{|c|c|c|c|c|c|c|c|}
\hline $\begin{array}{l}\text { Reference and } \\
\text { year }(\mathrm{m})\end{array}$ & $\begin{array}{l}\text { Number of } \\
\text { hexagonal } \\
\text { cell }\end{array}$ & $\begin{array}{l}\text { Inter-e } \\
\text { NodeB } \\
\text { distance }\end{array}$ & Distribution & $\begin{array}{l}\text { Speed } \\
\text { of users }\end{array}$ & Bandwidth & Strength & Weakness \\
\hline $\begin{array}{l}\text { (Manli et al., } \\
\text { 2015) }\end{array}$ & 21 & 1200 & Uniform & $0 \mathrm{Km} / \mathrm{h}$ & $20 \mathrm{MHz}$ & $\begin{array}{l}\text { This work has considered a } \\
\text { Cell- number }=21 \text { and a } \\
\text { Bandwidth }=20 \mathrm{MHz} \text { : } \\
\text { parameters compliant with those } \\
\text { of the } 4 \mathrm{G} \text { standard. }\end{array}$ & $\begin{array}{l}\text { To minimize ICI, this } \\
\text { work has increased inter- } \\
\text { eNodeB distance ( } 1.2 \\
\mathrm{Km} \text { ) and has considered } \\
\text { immobile users. }\end{array}$ \\
\hline $\begin{array}{l}\text { (Noor Shahida et al., } \\
\text { 2015) }\end{array}$ & 19 & 1000 & Normal & $3 \mathrm{Km} / \mathrm{h}$ & $20 \mathrm{MHz}$ & $\begin{array}{l}\text { This work has considered a } \\
\text { Bandwidth }=20 \mathrm{MHz} \text {. } \\
\text { distance }(1 \mathrm{Km}) \text { and has } \\
3 \mathrm{Km} / \mathrm{h} \text { (very low speed). } \\
\text { Cell- number (19) }\end{array}$ & $\begin{array}{l}\text { To minimize ICI, this work } \\
\text { has increased inter-eNodeB } \\
\text { considered users moving a } \\
\text { Also, this work has minimized }\end{array}$ \\
\hline $\begin{array}{l}\text { Hung-Chin and Wei-Di } \\
\text { (2015; Slawomir, 2017; } \\
\text { Sinh Cong et al., 2015; } \\
\text { Asif et al., 2017) }\end{array}$ & 21 & 2500 & Uniform & $0 \mathrm{Km} / \mathrm{h}$ & $20 \mathrm{MHz}$ & $\begin{array}{l}\text { This work has considered a } \\
\text { Cell-number }=21 \text { and a Bandwidth } \\
=20 \mathrm{MHz} \text { : parameters compliant } \\
\text { with those of the } 4 \mathrm{G} \text { standard. }\end{array}$ & $\begin{array}{l}\text { To minimize ICI, this work has } \\
\text { increased inter-eNodeB } \\
\text { distance }(2.5 \mathrm{Km}) \text { and has } \\
\text { considered immobile users. }\end{array}$ \\
\hline $\begin{array}{l}\text { (Slawomir } \\
\text { 2017) }\end{array}$ & 21 & 250 & Uniform & $0 \mathrm{Km} / \mathrm{h}$ & $20 \mathrm{MHz}$ & $\begin{array}{l}\text { This work has considered a } \\
\text { low inter-eNodeB distance } \\
(250 \mathrm{~m}<500 \mathrm{~m})\end{array}$ & $\begin{array}{l}\text { To minimize ICI, this work has } \\
\text { considered immobile users. }\end{array}$ \\
\hline $\begin{array}{l}\text { (Sinh Cong et al., } \\
\text { 2015) }\end{array}$ & 21 & 1000 & Uniform & $0 \mathrm{Km} / \mathrm{h}$ & $20 \mathrm{MHz}$ & $\begin{array}{l}\text { This work has considered a } \\
\text { Cell-number = } 21 \text { and a Bandwidth } \\
=20 \mathrm{MHz} \text { : parameters compliant } \\
\text { with those of the } 4 \mathrm{G} \text { standard. }\end{array}$ & $\begin{array}{l}\text { To minimize ICI, this work } \\
\text { has Increased inter-eNodeB } \\
\text { distance }(1 \mathrm{Km}) \text { and has } \\
\text { considered immobile users. }\end{array}$ \\
\hline $\begin{array}{l}\text { (Christos et al., } \\
\text { 2013) }\end{array}$ & 19 & 750 & Uniform & $0 \mathrm{Km} / \mathrm{h}$ & $20 \mathrm{MHz}$ & $\begin{array}{l}\text { This work has considered a } \\
\text { Bandwidth }=20 \mathrm{MHz}\end{array}$ & $\begin{array}{l}\text { To minimize ICI, this work } \\
\text { has increased inter-eNodeB } \\
\text { distance }(750 \mathrm{~m}) \text { and has } \\
\text { considered immobile users. } \\
\text { Also, this work has minimized } \\
\text { Cell-number (19) }\end{array}$ \\
\hline
\end{tabular}

Table 2: Simulation parameters

\begin{tabular}{ll}
\hline Parameters & Values \\
\hline Cell Layout & 3-Sectored hexagonal 7-cell cluster \\
Macro-cell Radius & $500 \mathrm{~m}$ \\
Number of Cells & 21 \\
Number of UEs & $10-200$ \\
Bandwidth & $20 \mathrm{MHz}$ \\
UEs Distribution & Randomly distributed moving at \\
& $300 \mathrm{Km} / \mathrm{h}$ \\
Transmit eNodeB Power & $46 \mathrm{dBm}$ \\
Transmit UE Power & $10 \mathrm{dBm}$ \\
\hline
\end{tabular}




\section{Simulation and Results}

After having configured the parameters of Table 1, we began the simulation of the Proposed Model (PM) while comparing it with SFR, DFFR and Strict FR techniques.

\section{A. SINR Performance in Uplink}

This scenario studied the SINR performance in uplink with a speed of $300 \mathrm{~km} / \mathrm{h}$. The number of users was varied between 10 and 200. The results of this scenario are described in Fig. 9. We note that the best method is the one that has the highest SINR (this corresponds to a low interference level).

Thus, the obtained results show that at $300 \mathrm{Km} / \mathrm{h}$ and for a users' number between 0 and 125 , the Strict FR method has the highest SINR and is therefore the best one for mitigating ICI in this case. But by increasing the users' number $(>125)$ and at $300 \mathrm{Km} / \mathrm{h}$, we notice that the best method is SFR. In this context, the model (PM) is better than both DFFR and Strict FR.

In addition and for the DFFR method, we notice that by increasing the number of users, the SINR decreases which explains the weak effect of this method in mitigating ICI in zones condensed in users.

With respect to this model, we note that by increasing the users' number $(>90)$, we remark that the SINR increases continuously, which explains the important effect of this model on ICI mitigation in condensed zones in users. In short, for condensed areas in mobile users, the two best ICI mitigation techniques are SFR and the model (PM).

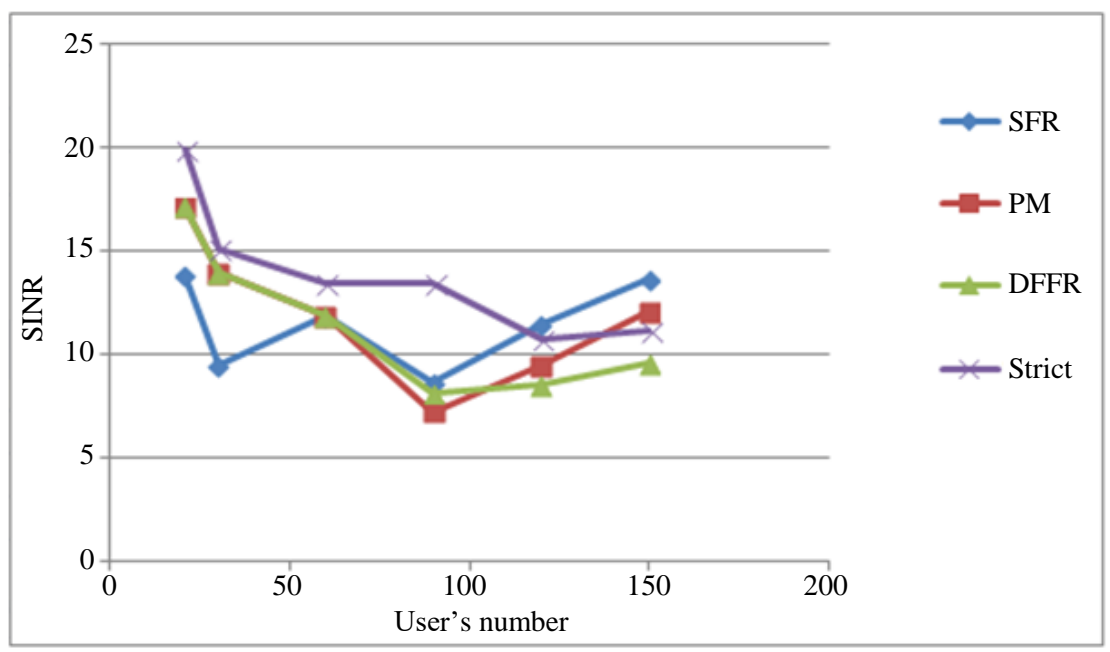

Fig. 9: SINR in uplink at $300 \mathrm{Km} / \mathrm{h}$

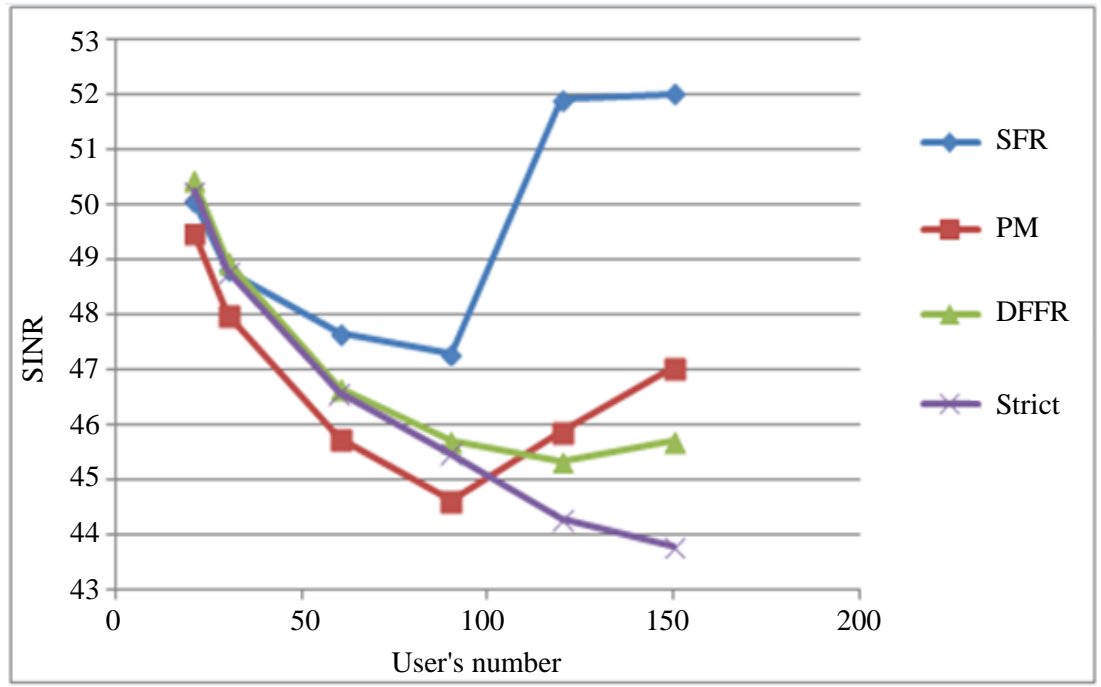

Fig. 10: SINR in downlink at $300 \mathrm{Km} / \mathrm{h}$ 
Emna Hajlaoui et al. / Journal of Computer Science 2019, 15 (12): 1820.1832 DOI: $10.3844 /$ jessp.2019.1820.1832

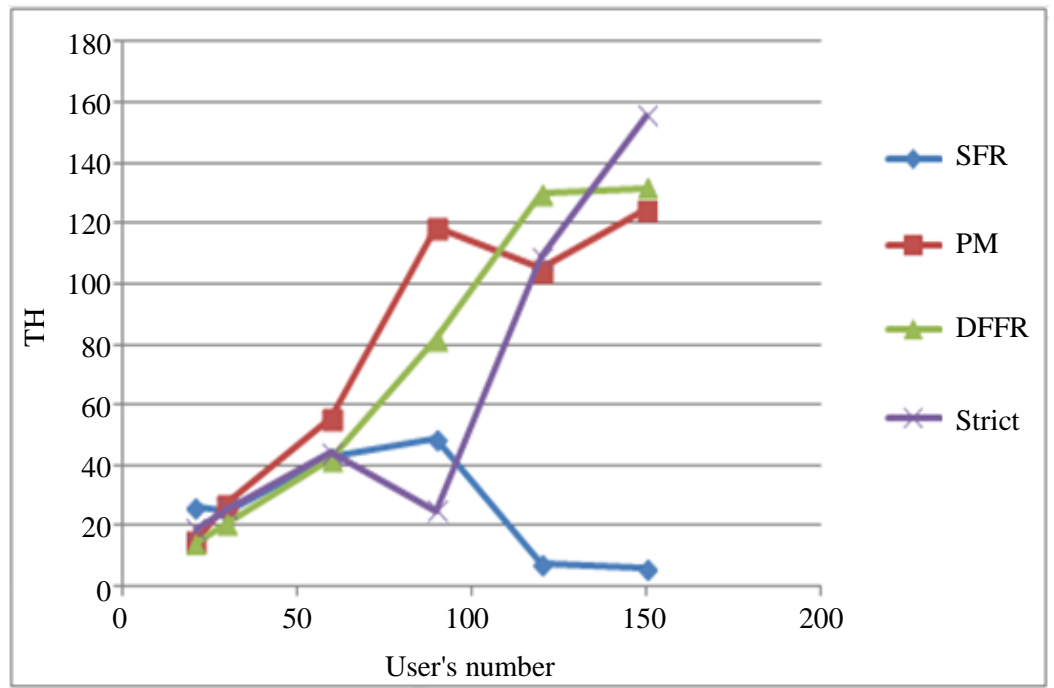

Fig. 11: Throughput in uplink at $300 \mathrm{Km} / \mathrm{h}$

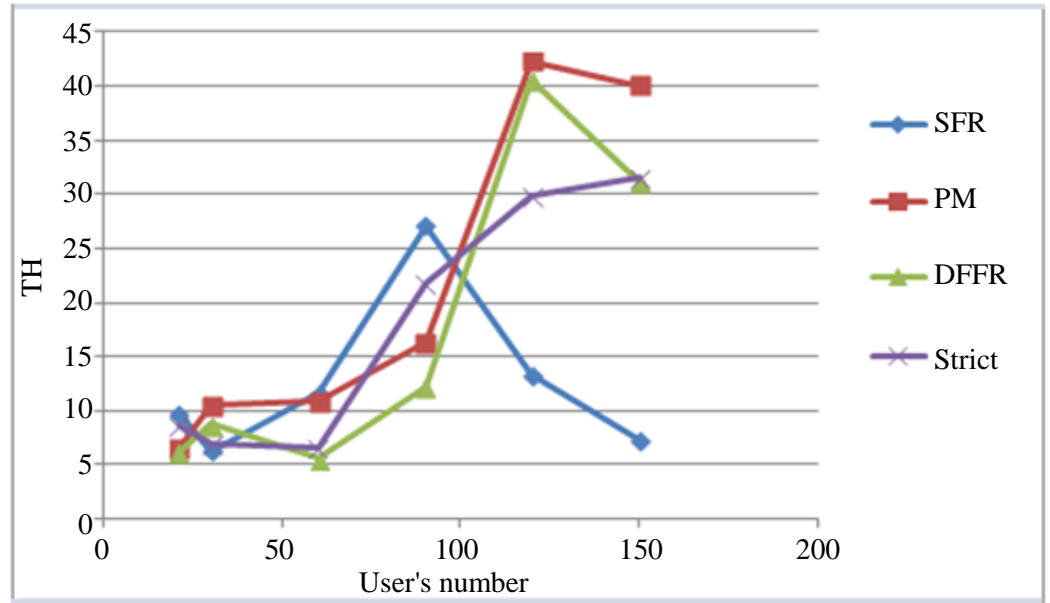

Fig. 12: Throughput in downlink at $300 \mathrm{Km} / \mathrm{h}$

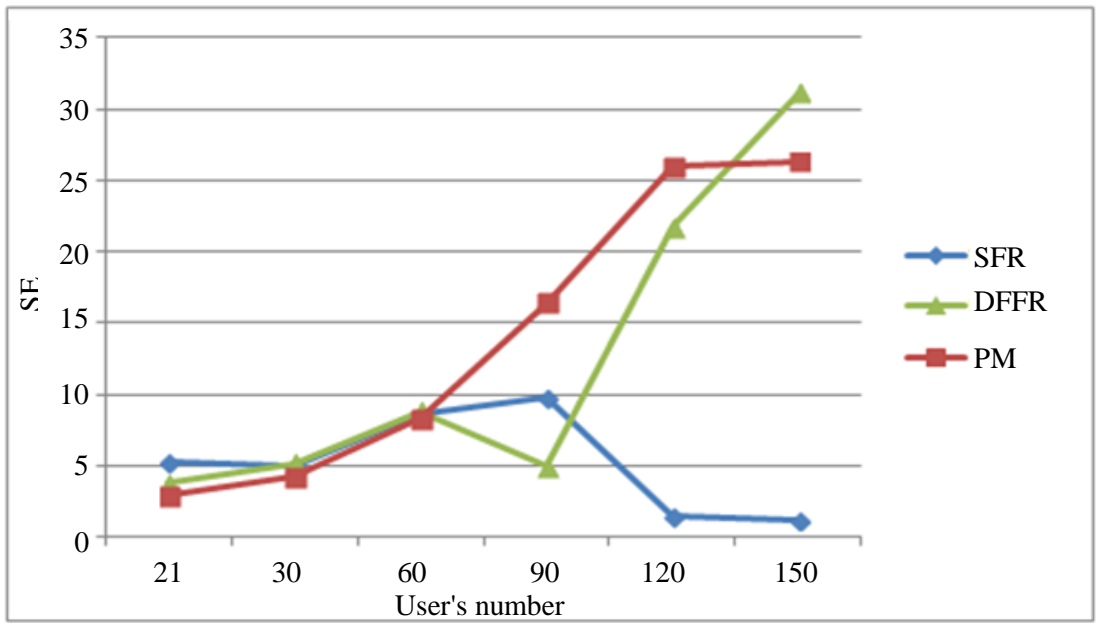

Fig. 13: Spectral efficiency in uplink at $300 \mathrm{Km} / \mathrm{h}$ 


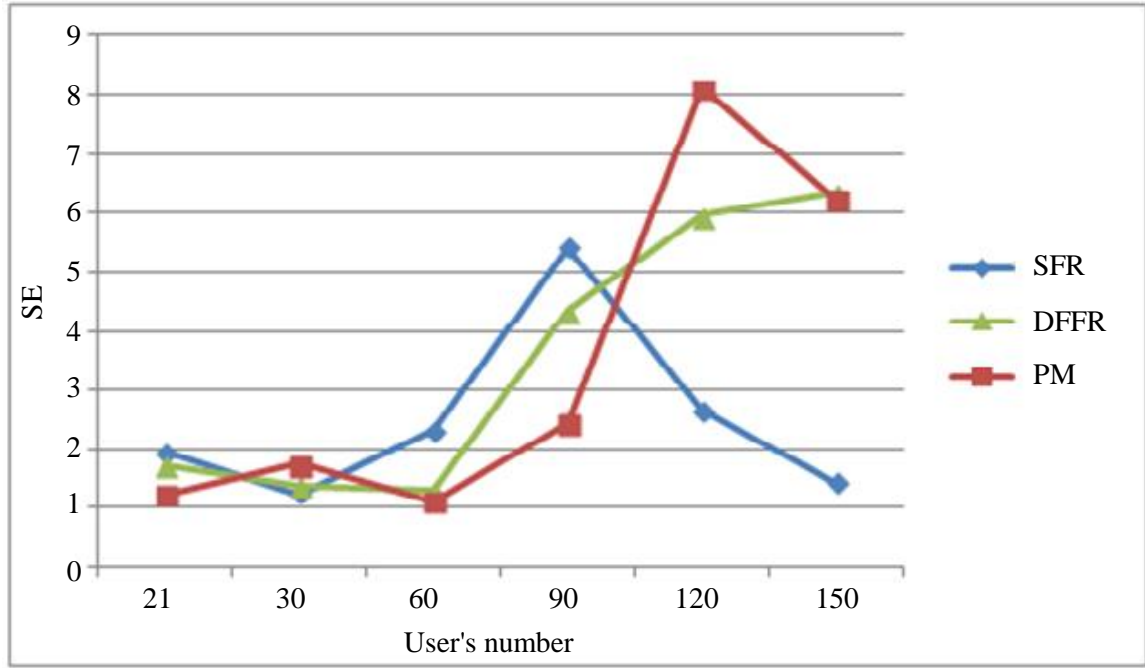

Fig. 14: Spectral efficiency in downlink at $300 \mathrm{Km} / \mathrm{h}$

\section{A. SINR Performance in Downlink}

This scenario studied the SINR performance in downlink with a speed of $300 \mathrm{~km} / \mathrm{h}$ and a number of users between 10 and 200. Results of this scenario are depicted in Fig. 10.

However, whatever the number of users and like in uplink, the SFR method is the most effective for ICI mitigation. This is due to the fact that users share the bandwidths with the neighboring cells.

For a large number of users (>120), we notice that the performances of the Proposed Model (PM) are better than those of DFFR and Strict FR methods.

To conclude, for both uplink and downlink and in condensed areas in mobile users (at $300 \mathrm{Km} / \mathrm{h}$ ), the best method for ICI mitigation is that SFR.

However, in both uplink and downlink and in areas condensed in mobile users (at $300 \mathrm{Km} / \mathrm{h}$ ), the proposed model has better performances than DFFR and Strict FR methods.

As a result, SFR method is effective in reducing ICI while increasing SINR in downlink.

\section{B. Throughput Performance in Uplink}

This scenario studied the throughput performance in uplink with a speed of $300 \mathrm{~km} / \mathrm{h}$. The number of users was varied between 10 and 200. Results of this scenario are described in Fig. 11. We note that the best method is the one that has the highest throughput.

Thus, the obtained results show that by increasing the users' number and at $300 \mathrm{Km} / \mathrm{h}$, the throughput increases continuously for the Proposed Model (PM), Strict FR and DFFR methods. However, we note that for a users' number $>90$, the throughput achieved by the SFR method decreases continuously and is close to zero for 150 users.
As a result, the proposed model, DFFR and Strict FR methods are successful at reducing ICI while increasing throughput in uplink.

\section{Throughput Performance in Downlink}

This scenario studied the throughput performance in downlink with a speed of $300 \mathrm{~km} / \mathrm{h}$. Number of users was varied between 10 and 200. Results of this scenario are presented in Fig. 12.

As in uplink, we notice that for users' number $>100$, the throughput of PM, DFFR and Strict FR methods increases continuously. Nevertheless, we note that for a users' number $>100$, the throughput achieved by the SFR method decreases continuously to achieve the lowest values for 150 users. However, for a condensed area in mobile users (> 105 users at $300 \mathrm{Km} / \mathrm{h}$ ), PM reaches the best performance in terms of throughput. Therefore, it is efficacious at reducing ICI while increasing throughput in downlink.

\section{Spectral Efficiency Performance in Uplink}

This scenario studied the spectral efficiency performance in uplink with a speed of $300 \mathrm{~km} / \mathrm{h}$. The number of users was varied between 10 and 200. Results of this scenario are illustrated in Fig. 13. We note that for a number of users between 21 and 60 , the results of the proposed model, SFR and DFFR methods are similar. Nevertheless, for a number of users between 60 and 130, this model (PM) achieves the best performance in terms of spectral efficiency. For users' number $>130$, DFFR method is the best one. Nevertheless, for users' number $>95$, the SFR method is the least effective in ICI mitigation. As a result, the PM and DFFR methods are efficient at reducing ICI while increasing spectral efficiency in uplink. 


\section{E. Spectral Efficiency Performance in Downlink}

This scenario studied the spectral efficiency performance in downlink with a speed of $300 \mathrm{~km} / \mathrm{h}$. The number of users was varied between 10 and 200. Results of this scenario are described in Fig. 14. We note that for a number of users between 21 and 30, the results of the proposed model, SFR and DFFR methods are similar. Nevertheless, for a number of users between 30 and 90, SFR method attains the best performance in terms of spectral efficiency. For users' number $>100$, the PM is the best one. However, for users' number $>100$, the SFR method is the least effective in ICI mitigation. As a result, the PM is efficacious at reducing ICI while increasing spectral efficiency in downlink.

\section{Conclusion}

4G users suffer from Intercell interference problem. Thus, in the literature, several techniques are proposed for mitigation of these interferences. In this context, well-known frequency reuse algorithms (SFR, DFFR and Strict FR) have been introduced as an effective way to optimize spectrum and control the ICI.

Thus, this study shows firstly, that the proposed model can provide acceptable performances in terms of SINR, throughput and cell spectral efficiency in the high-speed scenarios $(300 \mathrm{~km} / \mathrm{h})$ compared to previous works that evaluated and tested immobile scenarios. In this context and in terms of SINR in uplink and downlink traffics, we showed that for condensed area in mobile users, the two best ICI mitigation techniques are SFR and the model (PM). Moreover, it is shown that in terms of throughput, the proposed model, DFFR and Strict FR methods are efficient at reducing ICI while increasing throughput in uplink.

Furthermore, for a condensed area in mobile users (> 105 users at $300 \mathrm{Km} / \mathrm{h}$ ), the PM achieved the best performance in terms of throughput and spectral efficiency in downlink.

In short, in the different scenarios, the proposed model was always the best or the second best at reducing ICI, unlike the SFR scheme, which was always the least effective in mitigating ICI.

As a continuation of this work, we will try to test the effectiveness of this model with $5 \mathrm{G}$ wireless networks.

\section{Acknowledgment}

This work was supported by the Ministry of the Higher Education and Scientific Research in Tunisia.

\section{Author's Contributions}

Emna Hajlaoui: Participated in all experiments, coordinated the data collection and analysis and contributed to the writing of the manuscript.
Abdelhakim Khlifi: Validation of algorithms and implementation results.

Samia Dardouri: Concept development, data validation and contributed to the writing of the manuscript.

Aida Zaier: Concept development, preparation of journals and proofreading and contributed to the writing of the manuscript.

Mouna Ben Hamed: Contributed reviewing the article critically and gave the final approval of the version to be submitted.

Lassâad Sbita: Designed the research plan and organized the study

\section{Ethics}

This article is original and contains unpublished material. The authors testify that this paper submitted to the Journal of Computer Science has not been published elsewhere and that has no ethical issues.

\section{References}

3GPP TS 25.913, 2009. 3GPP, Tech, Specif. Group Radio access network requirements for evolved UTRA (E-UTRA) and Evolved UTRAN (EUTRAN), 3GPP TS 25.913.

Asif, R., B.A. Khaizuran, B.I. Ahmad Fadzil, R. Farah and B. Hudah Adibah et al., 2017. Dynamic frequency reuse: A method for interference mitigation in OFDMA based LTE-A networks. Int. J. Future Generat. Commun. Network., 10: 11-18.

Christos, B., D. Georgios, K. Vasileios, K. Konstantinos and P. Andreas, 2013. Wireless Personal Commun.

Dikamba, T., 2011. Downlink scheduling in 3GPP Long Term Evolution (LTE). MSc Thesis, TUDelft,

Ghassasn, A., A. Ismail and K. Jumari, 2012. The evolution to 4G cellular systems: Architecture and key features of LTE-advanced networks. Int. J. Comput. Networks Wireless Commun., 2: 2250-3501.

Giménez, S., D. Calabuig, F. Monserrat and N. Cardona, 2015. Dynamic and load-adapting distributed fractional frequency reuse algorithm for ultra-dense networks. Waves, 7: 1-8.

Harwahyu, R., Misbahuddin and R. Ftiri Sari, 2016. Performance evaluation of LTE random access procedure under distributed location data mining for road traffic monitoring. Int. J. Commun. Networks Inform. Security, 8: 171-1748.

Holma, H. and A. Toskala, 2009. LTE for UMTS: OFDMA and SC-FDMA Based Radio Access. 1st Edn., John Wiley and Sons, ISBN-10: 0470745479, pp: 450. 
Hung-Chin, J. and W. Wei-Di, 2015. Interference management using frequency reuse and CoMP for LTE-advanced networks. Proceedings of the 7 th International Conference on Ubiquitous and Future Networks, Jul. 7-10, IEEE Xplore Press, Sapporo, Japan, pp. 740-745.

DOI: 10.1109/ICUFN.2015.7182641

Hussain, S., 2009. Dynamic radio resource management in 3GPP LTE. School of Engineering, Department of Applied Signal Processing, Blekinge Institute of Technology.

Manli, Q., H. Wibowo, L. Yonghui, V. Branka and Y. Xuezhi et al., 2015. Adaptive soft frequency reuse scheme for wireless cellular networks. IEEE Trans. Vehicular Technol., 64: 118-131.

Noor Shahida, M.K., N. Rosdiadee and I. Mahamod, 2015. Power allocation for Dynamic Fractional Frequency Reuse (DFFR) in downlink LTE-A system. Proceedings of the 21st Asia-Pacific Conference on Communications, Oct. 14-16, IEEE Xplore Press, Kyoto, Japan, pp: 1-8.

DOI: 10.1109/APCC.2015.7412494

Pomilio de Oliveira, R., L. Aparecido de Gois and A. Foronda, 2018. Enhanced PF scheduling algorithm for LTE networks. Int. J. Commun. Networks Inform. Security, 10: 49-55.

Sadiq, B., B. Madan and R. Sampath, 2009. Downlink scheduling for multiclass traffic in LTE. EURASIP J. Wireless Commun. Network.
Sbit, S., B. Dadi and B. Chibani, 2018. Interference evaluation in cellular networks. Wireless Personal Commun., 100: 1299-1311. DOI: $10.1007 / \mathrm{s} 11277-018-5637-1$

Singh, D. and P. Singh, 2013. Radio resource scheduling in 3GPP LTE: A review. Int. J. Eng. Trends Technol., 4: 2405-2411.

Sinh Cong, L., S. Ramprasad, S. Kumbesan, G. Pantha and B. Shouman, 2015. Performance of well-known frequency reuse algorithms in LTE downlink 3GPP LTE systems. Proceedings of the 9th International Conference on Signal Processing and Communication Systems, Dec. 14-16, IEEE Xplore Press, Cairns, QLD, Australia. DOI: 10.1109/ICSPCS.2015.7391766

Slawomir, G., 2017. Soft-partial frequency reuse method for LTE-A. Radio Eng., 26: 359-368.

Veeresh, P., G. Sanjeev and C. Keshavamurthy, 2018. SRLNC based MAC optimization for multimedia data transmission over LTE/LTE-A network. Int. J. Commun. Networks Inform. Security, 10: 37-48.

Zaier, A., E. Hajlaoui and R. Bouallegue, 2016. Voice over LTE standards: Solutions and QoS control parameters in high mobile environments. Proceedings of the 30th International Conference on Advanced Information Networking and Applications Workshops, Mar. 23-25, IEEE Xplore Press, CransMontana, Switzerland, pp: 506-511.

DOI: 10.1109/WAINA.2016.132 